(2) Open Access Full Text Article

\title{
Impact of Cognitive Symptoms on Health-Related Quality of Life and Work Productivity in Chinese Patients with Major Depressive Disorder: Results from the PROACT Study
}

This article was published in the following Dove Press journal:

Neuropsychiatric Disease and Treatment

\author{
Gang Wang' \\ Kristin Hui Xian $\operatorname{Tan}^{2}$ \\ Hongye Ren ${ }^{3}$ \\ Lene Hammer-Helmich (iD ${ }^{3}$ \\ 'Psychiatry Department, Beijing an Ding \\ Hospital, Capital Medical University, \\ China National Clinical Research Center \\ for Mental Disorders, Beijing, People's \\ Republic of China; ${ }^{2}$ Health Economics \& \\ Epidemiology Statistics, Lundbeck \\ Singapore Pte Ltd, Singapore; ${ }^{3}$ Medical \\ Affairs Value Evidence, $H$. Lundbeck A/S, \\ Valby, Denmark
}

Purpose: This post hoc analysis was undertaken to further explore the association of cognitive symptoms with health-related quality of life (HRQoL) and work productivity at the time of treatment initiation in Chinese patients with major depressive disorder (MDD) in the Prospective Research Observation to Assess Cognition in Treated patients with MDD (PROACT) study.

Patients and Methods: This was an epidemiological, non-interventional, prospective cohort study in adult outpatients with moderate-to-severe MDD initiating antidepressant monotherapy (first or second line). Crude and adjusted analyses of covariance were performed to assess the association of perceived cognitive symptoms (20-item Perceived Deficits QuestionnaireDepression [PDQ-D] total score) or observed cognitive performance (Digit Symbol Substitution Test [DSST] score) with HRQoL (EuroQoL 5-Dimensions Questionnaire index) and work productivity (Work Productivity and Activity Impairment [WPAI] or Sheehan Disability Scale [SDS] absenteeism and presenteeism scores). Adjusted analyses included depression severity, age, sex, residential area (urban/rural), and educational level.

Results: Of 1008 patients enrolled in the PROACT study, 986 were included in this analysis. Severity of perceived cognitive symptoms (ie, higher PDQ-D total score) was significantly associated with worse HRQoL $(P<0.001)$ and higher levels of absenteeism $(P=0.020$ for the WPAI and $P=0.002$ for the SDS) and presenteeism ( $P<0.001$ for both scales). The association of perceived cognitive symptoms with HRQoL and presenteeism was independent of depression severity. The association between observed cognitive performance (DSST score) and HRQoL was less robust. No association was seen between observed cognitive performance and levels of absenteeism or presenteeism assessed by either scale.

Conclusion: Results of this real-world study illustrate the impact of cognitive symptoms on HRQoL and work productivity in Chinese patients with MDD, and highlight the importance of assessing and targeting cognitive symptoms in order to improve functional outcomes when treating patients with MDD.

Keywords: major depressive disorder, cognitive symptoms, health-related quality of life, work productivity, presenteeism, absenteeism

\section{Introduction}

Major depressive disorder (MDD) is a prevalent and disabling condition, accounting for $8.2 \%$ of years lived with disability worldwide. ${ }^{1}$ In China, weighted lifetime prevalence rates for depressive disorders and MDD were 6.8\% and 3.4\%, respectively, in $2013 .^{2}$ MDD is the second leading cause of years lived with disability in
Correspondence: Lene Hammer-Helmich Medical Affairs Value Evidence, $\mathrm{H}$. Lundbeck A/S, Ottiliavej 9, 2500 Valby, Denmark

Tel +4530832880

Email leem@lundbeck.com 
China; ${ }^{3}$ however, available data suggest that this condition is underdiagnosed and undertreated in Chinese populations. $^{4-6}$ In a recent cross-sectional study, only $8.3 \%$ of respondents meeting diagnostic criteria for MDD had been clinically diagnosed, and of these, only $51.5 \%$ were receiving antidepressant medication. ${ }^{6}$ Of note, Chinese adults with MDD reported significantly worse health-related quality of life (HRQoL) and greater productivity loss than those without depression. ${ }^{6}$

It is well documented that MDD imposes significant impairments in HRQoL and work productivity. ${ }^{7,8} \mathrm{MDD}$ is associated both with having to take time off work (absenteeism) and with reduced productivity when at work (presenteeism), ${ }^{9}$ and its impact on the ability to work and work productivity appears greater than that of other common mental and physical health disorders. ${ }^{10-12}$ Indeed, reduced work productivity accounts for the majority of the financial costs attributed to MDD. ${ }^{10,13}$

Cognitive symptoms are common in patients with MDD, with deficits observed across multiple domains (eg, executive function, memory, and attention). ${ }^{14-16}$ Such symptoms have been shown to negatively impact both clinical and functional outcomes in patients with MDD, including HRQoL and work productivity. ${ }^{17-28}$ However, data are lacking concerning the impact of cognitive symptoms on HRQoL and work productivity in Chinese patients with MDD. The Prospective Research Observation to Assess Cognition in Treated patients with MDD (PROACT) study was undertaken to evaluate the prevalence and course of cognitive symptoms in Chinese patients initiating new antidepressant treatment in a real-world setting. ${ }^{29}$ We present results of a post hoc analysis undertaken to further explore the association of cognitive symptoms with HRQoL and work productivity in the PROACT study population at the time of treatment initiation.

\section{Methods}

\section{Study Design}

PROACT was an epidemiological, non-interventional, prospective, cohort study conducted at 15 sites in China between March 2016 and July 2017. The study design has been reported in detail previously. ${ }^{29}$ Eligible participants were outpatients aged 18-65 years with a diagnosis of MDD (International Statistical Classification of Diseases and Related Health Problems [10th revision] criteria) and a total 17-item Hamilton Depression Rating Scale $\left(\mathrm{HAM}-\mathrm{D}_{17}\right)$ score $\geq 17$ (indicating moderate-to- severe depression), and were initiating antidepressant monotherapy (first- or second-line therapy). Key exclusion criteria included: comorbidities, including schizophrenia or other psychotic disorders, bipolar disorder, and alcohol or substance dependence; use of combination therapy (ie, receiving more than one antidepressant, adjunctive antipsychotics, or mood stabilizers); acute suicidality; and pregnancy/breastfeeding.

\section{Ethical Approval and Consent to Participate}

The study was conducted in accordance with the ethical principles described in the Declaration of Helsinki and the approved protocol, as well as all other applicable laws and regulations. Ethical approval was obtained from the necessary committees for each study site and all patients provided written informed consent for participation.

\section{Study Assessments}

Depression severity was evaluated by clinicians using the HAM-D ${ }_{17}$ and by patients using the 9-item Patient Health Questionnaire (PHQ-9). ${ }^{30,31}$ The HAM-D 17 assesses the severity of psychological and somatic depressive symptoms over the past week through a semi-structured interview. ${ }^{30}$ Each item is rated on a scale from 0 (absent) to 2 or 4 (maximum severity); individual item scores are then combined to yield a total score ranging from 0 to 52 . The PHQ-9 assesses the severity of depressive symptoms over the previous 2 weeks on a scale from 0 to $27 .{ }^{31}$ On both scales, higher scores indicate more severe depression.

Perceived cognitive symptoms were assessed by patients using the 20-item Perceived Deficits QuestionnaireDepression (PDQ-D). ${ }^{32-34}$ The PDQ-D assesses selfperceived difficulties over the previous 7 days across four domains of cognitive function: attention/concentration, prospective memory, retrospective memory, and planning/organization. Each of the 20 questions ( 5 per domain) is rated from 0 to 4 , yielding a possible total score of 0 to 80 ; higher scores indicate greater perceived cognitive symptoms. The Chinese version of the PDQ-D has been shown to be psychometrically valid for the evaluation of subjective cognitive symptoms in patients with MDD. ${ }^{33}$

Cognitive performance was evaluated using the Digit Symbol Substitution Test (DSST), a neuropsychological coding test that requires the patient to substitute simple symbols for digits. ${ }^{35}$ The number of correct symbols substituted for digits during a 90 -second period provides 
a score ranging from 0 to 133 , with higher scores reflecting better cognitive performance. The DSST assesses cognitive performance across a number of domains, including those known to be impaired in patients with MDD, such as executive function, processing speed and attention. The DSST has shown sensitivity to change in MDD populations, ${ }^{17,36,37}$ and age-related norms have been defined for US adults. ${ }^{35}$

HRQoL was assessed with the widely used self-reported EuroQoL 5-Dimensions Questionnaire (EQ-5D). ${ }^{38}$ This instrument encompasses five health dimensions (mobility, self-care, usual activities, pain/discomfort, and anxiety/ depression). Responses were recorded using three levels of severity (no problem, some problems, or extreme problems). The EQ-5D assesses HRQoL at the time of completion; there is no recall period. The EQ-5D utility index score was computed, based on coefficients derived from regression results in a Chinese population. ${ }^{39} \mathrm{An}$ EQ-5D utility index score of 1 indicates perfect health, 0 indicates a state equivalent to being dead, and $<0$ indicates a state considered worse than being dead.

Work productivity was assessed by the Work Productivity and Activity Impairment - Specific Health Problems Questionnaire (WPAI-SHP) ${ }^{40}$ and the Sheehan Disability Scale (SDS). ${ }^{41,42}$ The WPAI questionnaire is a validated self-reported instrument for the assessment of work-related impairment due to a health problem such as MDD over the past 7 days. $^{40}$ The WPAI measures work hours missed due to the health problem, work hours missed due to other reasons, hours worked, the degree to which the health problem affected productivity while working, and the degree to which the health problem affected non-work daily activities. Based on validated algorithms, the WPAI yields four subscores: absenteeism (percent work time missed), presenteeism (percent time impaired while working), work productivity loss (percent overall work impairment), and percent overall activity impairment. Higher scores indicate greater impairment and lower work productivity.

The SDS is a brief self-administered tool that evaluates functional impairment during the previous 7 days across three domains: work/school, social life/leisure activities, and family life/home duties. ${ }^{41,42}$ The severity of impairment in each domain is rated by the patient on a scale from 0 (no impairment) to 10 (extreme impairment). The SDS also assesses the number of work/study days lost (absenteeism) and the number of underproductive work/study days (presenteeism) over the past week.

\section{Statistical Analysis}

The population for this analysis comprised all enrolled patients who complied with the study inclusion and exclusion criteria. Crude and adjusted analyses of covariance were performed to assess the association of perceived cognitive symptoms (PDQ-D total score) or observed cognitive performance (DSST total score) with HRQoL (EQ5D utility index) and work productivity (WPAI or SDS absenteeism and presenteeism scores). Patients were categorized into groups based on quartiles of PDQ-D total score at study entry: (a) 0-20; (b) 21-32; (c) 33-44; and (d) 45-80. For DSST, patients were categorized into four mutually exclusive categories based on DSST total score and age at baseline: (a) within norm; (b) $1 / 3-2 / 3$ standard deviation (SD) below norm; (c) $2 / 3-1$ SD below norm; and (d) $\geq 1$ SD below norm. As DSST norms were developed using data from US adults, ${ }^{35}$ patients were also categorized into groups based on DSST total score quartiles at baseline: (a) 0-37; (b) 38-51; (c) 52-62; and (d) 63-133. For this analysis, lost work days and underproductive work days were disregarded if patients indicated that they had not worked or studied at all during the past week for reasons unrelated to MDD. In the adjusted analyses, depression severity (PHQ-9 or HAM-D 17 score) was adjusted as a continuous variable, together with age, sex, residential area (urban/rural), and educational level, as these were identified as possible confounders based on clinical experience and in line with the primary analysis of data from this study. ${ }^{29}$ All analyses were performed using $\mathrm{R}$ version 3.5.1. ${ }^{43}$

\section{Results}

Of 1008 patients enrolled in the PROACT study, 986 were included in this analysis (97.8\%). Reasons for exclusion from the study, and hence this analysis population, were: not initiating antidepressant monotherapy at baseline $(n=14)$; use of antipsychotics or mood stabilizers at baseline $(n=7)$; pregnant or $\leq 6$ months postpartum $(n=12)$; and unable to read and/or understand the information sheet and informed consent form $(n=2)$. Patients could have had more than one reason for exclusion.

Patient demographics and clinical characteristics are shown in Table 1. Mean (SD) age was 35.9 (12.0) years, and patients were mostly female $(68.5 \%)$, lived in urban areas $(82.2 \%)$, and had received formal education up to at least high school level (76.7\%). Just over half of the study participants were employed (57.0\%). Patients were 
Table I Baseline Patient Demographics and Clinical Characteristics

\begin{tabular}{|c|c|}
\hline Characteristic & $\begin{array}{l}\text { Patients with MDD } \\
(n=986)\end{array}$ \\
\hline Age (years), mean (SD) & $35.9(12.0)$ \\
\hline Female sex, \% (n) & $68.5(675)$ \\
\hline \multicolumn{2}{|l|}{ Region in China, \% (n) } \\
\hline North & $56.9(56 I)$ \\
\hline South & $6.8(67)$ \\
\hline East & $17.5(173)$ \\
\hline West & $18.8(185)$ \\
\hline \multicolumn{2}{|l|}{ Residential area, \% (n) } \\
\hline Urban & $82.2(810)$ \\
\hline Rural & I7.8 (I76) \\
\hline Employed, \% (n) & $57.0(562)$ \\
\hline \multicolumn{2}{|l|}{ Educational level, \% (n) } \\
\hline Middle school or lower & $23.3(230)$ \\
\hline High school or junior college & $31.7(3 \mid 3)$ \\
\hline University or postgraduate & $44.9(443)$ \\
\hline \multicolumn{2}{|l|}{ Clinical history, \% (n) } \\
\hline Previous episode & $39.2(387)$ \\
\hline Switching antidepressant at baseline & $20.6(203)$ \\
\hline \multicolumn{2}{|l|}{$\begin{array}{l}\text { Baseline assessment scores, } \\
\text { mean (SD) }\end{array}$} \\
\hline HAM-D ${ }_{17}$ total score & $23.2(4.4)$ \\
\hline PHQ-9 total score & $16.7(5.8)$ \\
\hline DSST & $50.7(16.8)$ \\
\hline PDQ-D total score & $33.6(16.3)$ \\
\hline EQ-5D utility index & $0.74(0.14)$ \\
\hline SDS absenteeism (work days lost) & $2.0(2.5)$ \\
\hline $\begin{array}{l}\text { SDS presenteeism (work days } \\
\text { underproductive) }\end{array}$ & $3.7(2.5)$ \\
\hline WPAI absenteeism (\%) & $26.3(35.7)$ \\
\hline WPAI presenteeism (\%) & $54.5(26.8)$ \\
\hline \multicolumn{2}{|l|}{ PDQ-D quartile, \% (n) } \\
\hline $0-20$ & $24.0(23 I)$ \\
\hline $21-32$ & $25.7(247)$ \\
\hline $33-44$ & $24.9(239)$ \\
\hline $45-80$ & $25.4(244)$ \\
\hline Missing (n) & 25 \\
\hline \multicolumn{2}{|l|}{ DSST subgroup, \% (n) } \\
\hline Within norm & $45.4(445)$ \\
\hline $1 / 3-2 / 3$ SD below norm & $13.3(130)$ \\
\hline $2 / 3-1$ SD below norm & $10.9(107)$ \\
\hline$\geq$ I SD below norm & 30.5 (299) \\
\hline Missing (n) & 5 \\
\hline
\end{tabular}

(Continued)
Table I (Continued).

\begin{tabular}{|l|l|}
\hline Characteristic & $\begin{array}{l}\text { Patients with MDD } \\
(\mathbf{n = 9 8 6 )}\end{array}$ \\
\hline DSST quartile, \% (n) & \\
$0-37$ & $23.8(233)$ \\
$38-51$ & $25.0(245)$ \\
$52-62$ & $25.9(254)$ \\
$63-133$ & $25.4(249)$ \\
Missing (n) & 5 \\
\hline
\end{tabular}

Abbreviations: DSST, Digit Symbol Substitution Test; EQ-5D, EuroQol 5-Dimensions Questionnaire; HAM-D ${ }_{17}, 17$-item Hamilton Depression Rating Scale; MDD, major depressive disorder; PDQ-D, 20-item Perceived Deficits Questionnaire-Depression; PHQ-9, 9-item Patient Health Questionnaire; SD, standard deviation; SDS, Sheehan Disability Scale; WPAI, Work Productivity and Activity Impairment.

predominantly initiating first-line antidepressant treatment (79.4\%). Mean (SD) HAM-D 17 total score was 23.2 (4.4) and mean (SD) PHQ-9 total score was 16.7 (5.8). Over half of all patients $(54.6 \%)$ had DSST score $\geq 1 / 3$ SD below norm. Mean (SD) DSST score was 50.7 (16.8) and mean (SD) PDQ-D total score was 33.6 (16.3). With regard to impairment in HRQoL and work productivity, mean (SD) EQ-5D utility index score at study entry was $0.74(0.14)$, mean (SD) WPAI absenteeism and presenteeism scores were $26.3 \%(35.7 \%)$ and $54.5 \%(26.8 \%)$, respectively, and the mean (SD) number of working days during the past week that were lost or unproductive, as assessed using the SDS, were 2.0 (2.5) and 3.7 (2.5), respectively.

Results of the analyses performed to assess the association of perceived cognitive symptoms (PDQ-D score) with HRQoL (EQ-5D utility index) and work productivity (WPAI or SDS absenteeism and presenteeism scores) are shown in Table 2. Higher PDQ-D total score (ie, greater severity of perceived cognitive symptoms) was found to be associated with both lower HRQoL $(P<0.001)$ and higher levels of absenteeism ( $P=0.020$ for the WPAI and $P=0.002$ for the SDS) and presenteeism ( $P<0.001$ for both scales). The association of PDQ-D total score with HRQoL and presenteeism was shown to be independent of depression severity, as it remained statistically significant when the analysis was adjusted for PHQ-9 or HAM-D ${ }_{17}$ scores. However, the association between PDQ-D total score and absenteeism did not persist when adjusted for PHQ-9 or HAM-D ${ }_{17}$ scores, suggesting that this was largely driven by depression severity. 


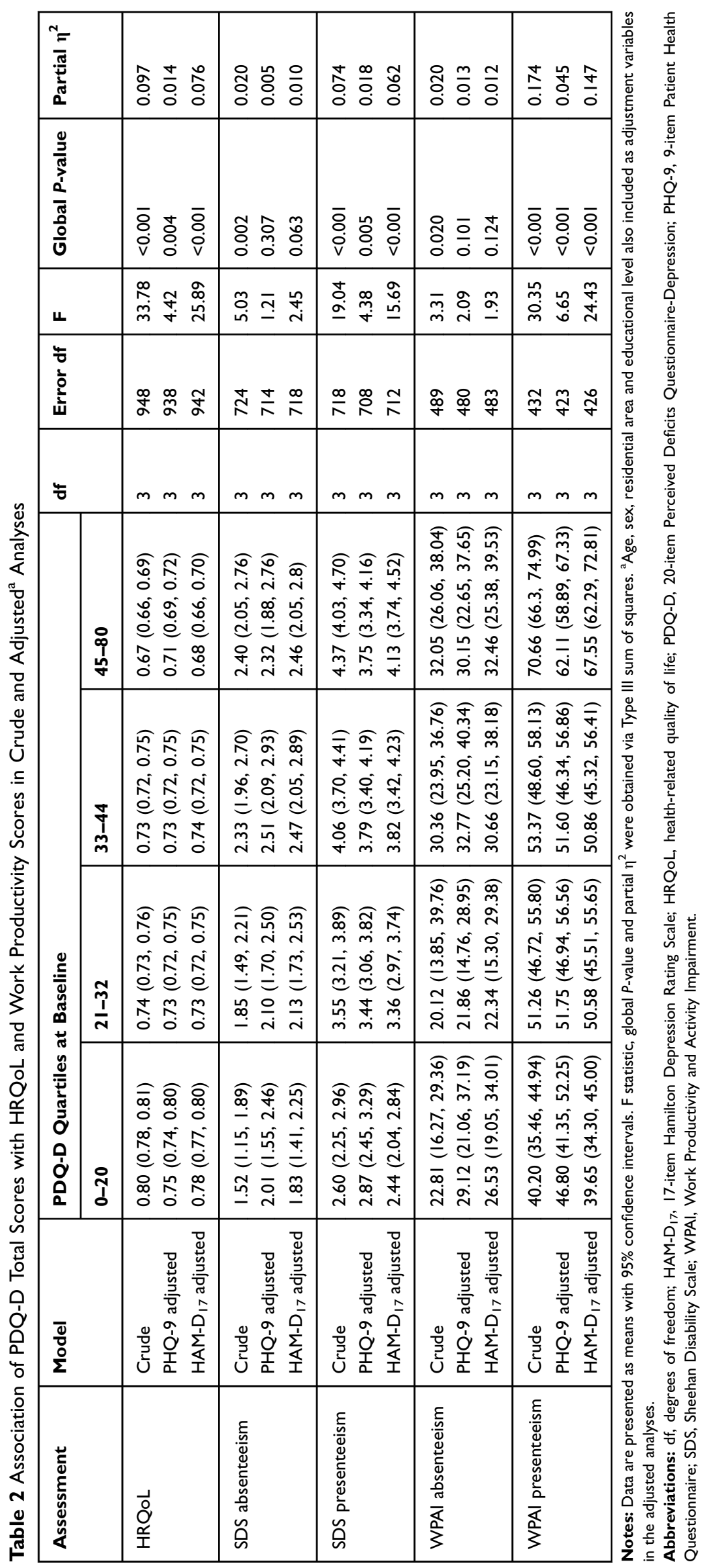




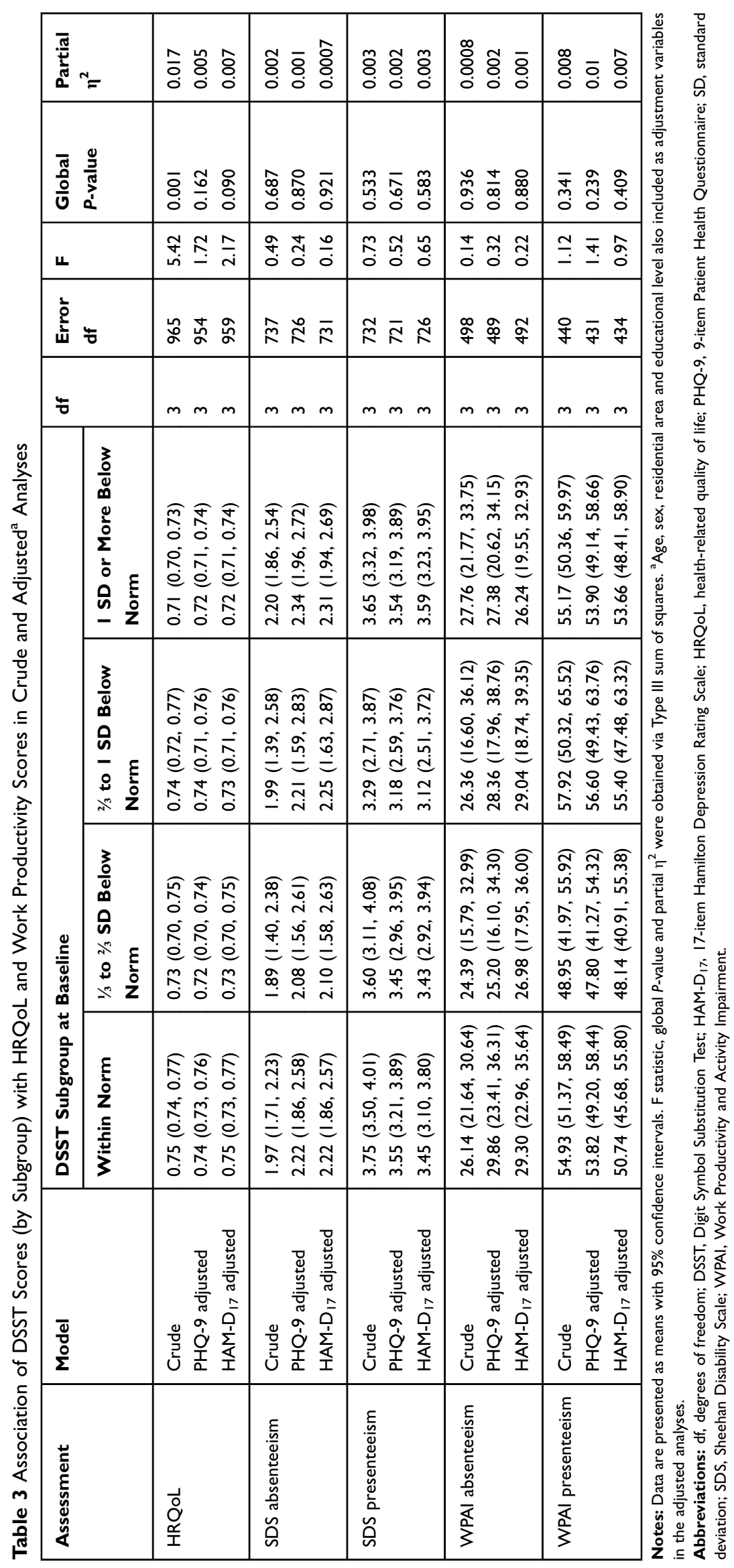




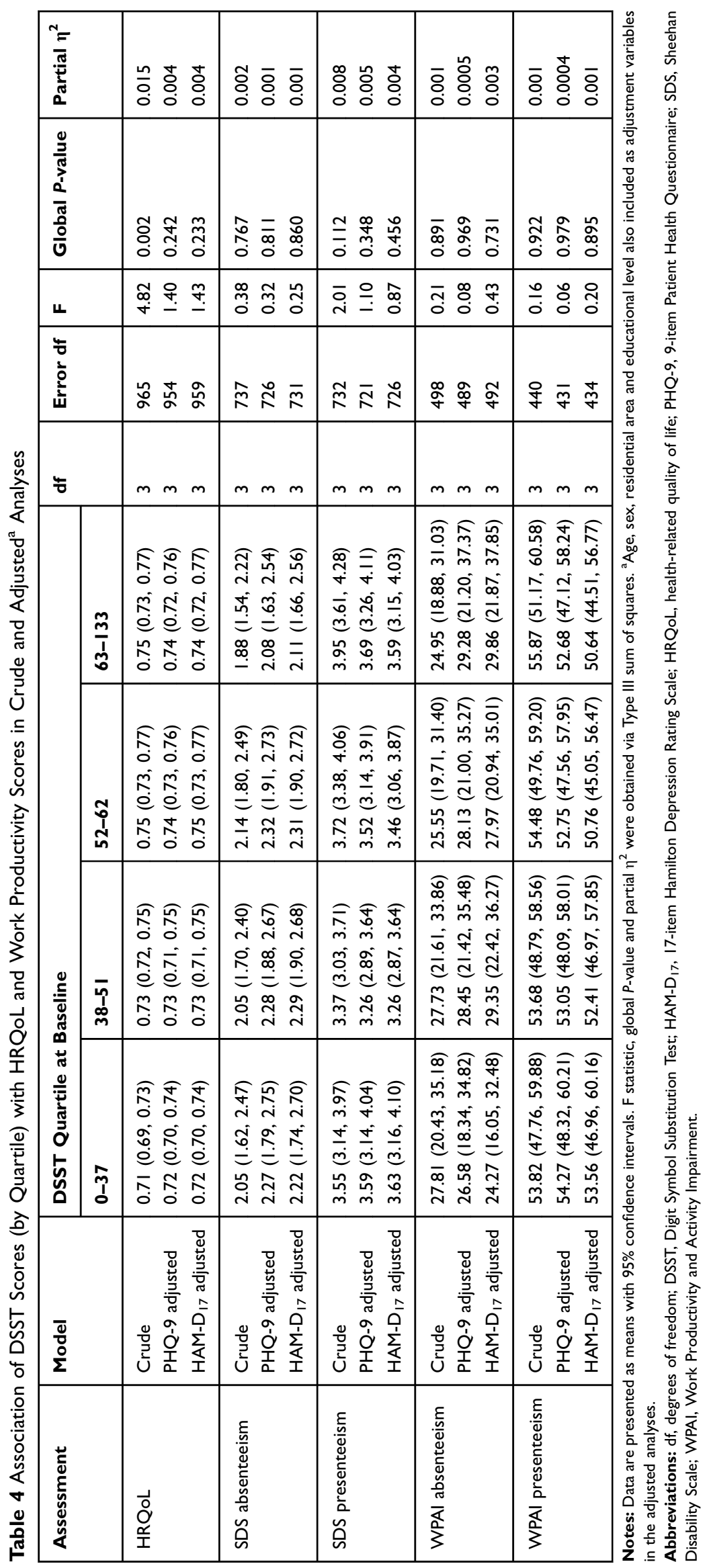


Results of the analyses performed to assess the association of cognitive performance (DSST score) with HRQoL (EQ-5D utility index) and work productivity (WPAI or SDS absenteeism and presenteeism scores) are shown in Tables 3 and 4. Lower DSST total score (ie, lower cognitive performance) was associated with lower HRQoL ( $P=0.001$ by DSST subgroup and $P=0.002$ by DSST quartile). However, the association between DSST total score and HRQoL did not persist when adjusted for PHQ-9 or HAM-D 17 scores. No association was seen between DSST total score and levels of absenteeism or presenteeism assessed by either scale.

\section{Discussion}

To our knowledge, this is the first study to describe the impact of cognitive symptoms on HRQoL and work productivity in Chinese patients with MDD. Patients were required to have moderate-to-severe depression to be eligible for study entry and most were initiating first-line antidepressant treatment. Most patients reported cognitive symptoms and over half were shown to have impaired cognitive performance by DSST. Mean DSST score at the time of treatment of initiation in this study (50.7) was similar to that reported in Japanese patients initiating new antidepressant therapy in another recent study (54.3), ${ }^{44}$ but higher than those reported in comparable US and European populations (42.0 in FOCUS and 43.1 in CONNECT). ${ }^{36,45}$ This may at least partly reflect cultural differences between the different geographic populations. Levels of impairment of HRQoL and work productivity were consistent with those reported in studies in similar MDD populations in other countries. ${ }^{18,20,21,23-25}$ Our findings also suggest that HRQoL may be more impaired by MDD than by other common chronic diseases in Chinese patients. The reported mean EQ-5D utility index score in this study was lower than reported for Chinese patients with other chronic health conditions, such as diabetes, hypertension, and epilepsy, ${ }^{46}$ and similar to those reported for Chinese patients with a range of cancer types. ${ }^{47}$

Patients with more severe perceived cognitive symptoms were shown to experience greater impairments in HRQoL and work productivity (absenteeism and presenteeism), even after adjustment for depression severity. In contrast, less robust correlations were seen between observed cognitive performance (assessed using the DSST) and HRQoL, with no associations seen between DSST and work productivity scores. This finding is not unexpected, as several other studies have also found discrepancies between self-reported measures and performance tests of cognitive symptoms in patients with MDD..$^{25,26,48-51}$ For example, in a study in the United States involving 602 patients with MDD reporting cognitive symptoms, 64\% met criteria for objectively defined cognitive impairment assessed using a battery of neuropsychological tests (including the DSST) and $48 \%$ met criteria for subjectively defined cognitive impairment assessed using the Cognitive and Physical Functioning Questionnaire (CPFQ). ${ }^{50}$ Overall, $80 \%$ of patients met criteria for either subjective or objective cognitive impairment; however, only $31 \%$ met both criteria.

The observed dissonance between objective and subjective cognitive performance in patients with MDD may be due to differences in the cognitive subdomains assessed by the various questionnaires and tests. There is also evidence to suggest differential effects of potential moderating factors. For example, data from the Cognitive Dysfunction in Asians with Depression (CogDAD) study show depression severity to be predictive of subjective cognitive performance, but not objective measures of cognition. ${ }^{49}$ From a clinical perspective, subjective and objective methods for assessing cognition complement each other and both methods are valuable. While the potential for bias cannot be excluded with self-assessment of cognitive symptoms, it is possible that subjective assessment of cognitive symptoms may more closely reflect the degree of impairment experienced by patients in daily life than objective assessment in a highly standardized test setting. ${ }^{52}$

As in other recent studies in patients with depression, ${ }^{20,23,25,26,53,54}$ presenteeism was found to be more prevalent than absenteeism in the study population. Patients reported missing an average of 2 working days during the previous week and over half of their time spent at work was deemed unproductive (3.7 days). Mean WPAI absenteeism and presenteeism scores in this study $(26.3 \%$ and $54.5 \%$ ) were considerably higher than those reported in a US study of patients with depression initiating antidepressant treatment $(8.2 \%$ and $35.2 \%){ }^{55}$ MDD-related work impairment has been shown to be associated with substantial economic costs. ${ }^{8,53,56}$

Coupled with the results from the primary analysis of the PROACT study, in which structural equation modelling showed severity of cognitive symptoms to consistently predict both clinical and functional outcomes over a 6-month period independent of depressive symptom severity, ${ }^{29}$ our findings suggest that cognitive symptoms 
should be considered an important and independent treatment target in Chinese patients with MDD. Results of a recent meta-analysis suggest differential effects of currently available antidepressants in terms of improving cognitive symptoms in patients with MDD. ${ }^{57}$

A major strength of the PROACT study is that it was performed in a large and well-defined population of Chinese patients with MDD in a real-world setting; hence, findings should be generalizable to routine practice. In addition, patient-reported outcome measures were used to assess disease severity and impact on HRQoL and work productivity from the patient's own perspective; this is known to be particularly valid in patients with mental health disorders such as MDD. ${ }^{58}$ A potential limitation is that the study population mainly comprised well-educated, urban residents initiating first- or second-line antidepressant monotherapy, which may limit generalization of the study findings to other groups of patients. Furthermore, as norms are not available for Chinese patients, age-related norms for US adults were used in the DSST subgroup analyses described in this paper. However, to address this potential limitation, DSST analyses were also performed according to DSST total-score quartile at baseline.

In summary, results of this real-world study illustrate the impact of cognitive symptoms on HRQoL and work productivity in Chinese patients with MDD, and highlight the importance of assessing and targeting cognitive symptoms in order to improve functional outcomes when treating patients with MDD.

\section{Abbreviations}

DSST, Digit Symbol Substitution Test; EQ-5D, EuroQoL 5-Dimensions Questionnaire; HAM-D 17 , 17-item Hamilton Depression Rating Scale; HRQoL, health-related quality of life; MDD, major depressive disorder; PDQ-D, Perceived Deficits Questionnaire-Depression; PHQ-9, 9-item Patient Health Questionnaire; PROACT, Prospective Research Observation to Assess Cognition in Treated patients with MDD; SD, standard deviation; SDS, Sheehan Disability Scale; WPAI-SHP, Work Productivity and Activity Impairment - Specific Health Problems Questionnaire.

\section{Data Sharing Statement}

The authors declare that the data relating to the reported analyses are available within the article. H. Lundbeck A/S may be contacted for further data sharing, including individual-level data.

\section{Acknowledgments}

The authors thank all the patients who participated in this study, as well as the investigators and study personnel at the following sites: Beijing An Ding Hospital, Capital Medical University; No. 6 Hospital of Beijing University; Shanghai Mental Health Centre, Shanghai Jiao Tong University School of Medicine; Second Xiangya Hospital of Central South University; West China Hospital, Sichuan University; Nanjing Brain Hospital; Beijing Huilongguan Hospital; Shenzhen Mental Health Center; First Affiliated Hospital of Kunming Medical College; Tianjin Anding Hospital; Beijing Tian Tan Hospital; First Affiliated Hospital of Medical College of Xi'an Jiaotong University; Beijing Chaoyang Hospital; Third People's Hospital of Huzhou City; and Third Hospital of Sun Yat-Sen University. Writing and editorial assistance was provided by Jennifer Coward of Anthemis Consulting Ltd, funded by $\mathrm{H}$. Lundbeck A/S. The PROACT study was sponsored by H. Lundbeck A/S and Lundbeck China.

\section{Author Contributions}

$\mathrm{GW}, \mathrm{HR}$ and $\mathrm{LH}-\mathrm{H}$ were involved in conceptualization and design of the study, GW was involved in the acquisition of data, TKHX was responsible for data analysis, and all authors contributed to data interpretation. The authors are entirely responsible for the scientific content of this paper and all authors contributed to drafting or revising the article, gave final approval of the version to be published, and agree to be accountable for all aspects of the work.

\section{Disclosure}

GW has received honoraria for being an advisor to or providing educational talks for Lundbeck, Pfizer, Sumitomo, Johnson \& Johnson, and Eli Lilly and Company. TKHX was a full-time employee of Lundbeck Singapore Pte Ltd when the study and analyses were performed. $\mathrm{HR}$ and $\mathrm{LH}-\mathrm{H}$ are full-time employees of H. Lundbeck A/S. The authors report no other conflicts of interest in this work.

\section{References}

1. Ferrari AJ, Charlson FJ, Norman RE, et al. Burden of depressive disorders by country, sex, age, and year: findings from the global burden of disease study 2010. PLoS Med. 2013;10(11):e1001547. doi:10.1371/journal.pmed.1001547

2. Huang Y, Wang Y, Wang H, et al. Prevalence of mental disorders in China: a cross-sectional epidemiological study. Lancet Psychiatry. 2019;6(3):211-224. doi:10.1016/S2215-0366(18)30511-X 
3. Yang G, Wang Y, Zeng Y, et al. Rapid health transition in China, 1990-2010: findings from the Global Burden of Disease Study 2010. Lancet. 2013;381(9882):1987-2015. doi:10.1016/S0140-6736(13) 61097-1

4. Phillips MR, Zhang J, Shi Q, et al. Prevalence, treatment, and associated disability of mental disorders in four provinces in China during 2001-05: an epidemiological survey. Lancet. 2009;373(9680): 2041-2053. doi:10.1016/S0140-6736(09)60660-7

5. Lao CK, Chan YM, Tong HH, Chan A. Underdiagnosis of depression in an economically deprived population in Macao, China. Asia Pac Psychiatry. 2016;8(1):70-79. doi:10.1111/appy.12208

6. Gupta S, Goren A, Dong P, Liu D. Prevalence, awareness, and burden of major depressive disorder in urban China. Expert Rev Pharmacoecon Outcomes Res. 2016;16(3):393-407. doi:10.1586/147 37167.2016.1102062

7. IsHak WW, Greenberg JM, Balayan K, et al. Quality of life: the ultimate outcome measure of interventions in major depressive disorder. Harv Rev Psychiatry. 2011;19(5):229-239. doi:10.3109/ 10673229.2011.614099

8. Woo J-M, Kim W, Hwang T-Y, et al. Impact of depression on work productivity and its improvement after outpatient treatment with antidepressants. Value Health. 2011;14(4):475-482. doi:10.1016/j. jval.2010.11.006

9. Birnbaum HG, Kessler RC, Kelley D, Ben-Hamadi R, Joish VN, Greenberg PE. Employer burden of mild, moderate, and severe major depressive disorder: mental health services utilization and costs, and work performance. Depress Anxiety. 2010;27(1):78-89. doi:10.1002/da.20580

10. Stewart WF, Ricci JA, Chee E, Hahn SR, Morganstein D. Cost of lost productive work time among US workers with depression. JAMA. 2003;289(23):3135-3144. doi:10.1001/jama.289.23.3135

11. de Graaf R, Tuithof M, van Dorsselaer S, Ten Have M. Comparing the effects on work performance of mental and physical disorders. Soc Psychiatry Psychiatr Epidemiol. 2012;47(11):1873-1883. doi:10.1007/s00127-012-0496-7

12. Allen D, Hines EW, Pazdernik V, Konecny LT, Breitenbach E. Fouryear review of presenteeism data among employees of a large United States health care system: a retrospective prevalence study. Hum Resour Health. 2018;16(1):59. doi:10.1186/s12960-018-0321-9

13. Ekman M, Granström O, Omérov S, Jacob J, Landén M. The societal cost of depression: evidence from 10,000 Swedish patients in psychiatric care. J Affect Disord. 2013;150(3):790-797. doi:10.1016/j.jad.2013.03.003

14. Evans VC, Iverson GL, Yatham LN, Lam RW. The relationship between neurocognitive and psychosocial functioning in major depressive disorder: a systematic review. J Clin Psychiatry. 2014;75 (12):1359-1370. doi:10.4088/JCP.13r08939

15. Lam RW, Kennedy SH, Mclntyre RS, Khullar A. Cognitive dysfunction in major depressive disorder: effects on psychosocial functioning and implications for treatment. Can J Psychiatry. 2014;59 (12):649-654. doi:10.1177/070674371405901206

16. Knight MJ, Baune BT. Cognitive dysfunction in major depressive disorder. Curr Opin Psychiatry. 2018;31(1):2631. doi:10.1097/YCO. 0000000000000378

17. McIntyre RS, Cha DS, Soczynska JK, et al. Cognitive deficits and functional outcomes in major depressive disorder: determinants, substrates, and treatment interventions. Depress Anxiety. 2013;30 (6):515-527. doi:10.1002/da.22063

18. Srisurapanont M, Bautista D, Chen CH, Wang G, Udomratn P, Eurviriyanukul K. Subjective memory and concentration deficits in medication-free, non-elderly Asians with major depressive disorder: prevalence and their correlates. J Affect Disord. 2015;171:105-110. doi:10.1016/j.jad.2014.09.007

19. Clark M, DiBenedetti D, Perez V. Cognitive dysfunction and work productivity in major depressive disorder. Expert Rev Pharmacoecon Outcomes Res. 2016;16(4):455-463. doi:10.1080/14737167.2016.119 5688
20. Kim JM, Chalem Y, Di Nicola S, Hong JP, Won SH, Milea D. A cross-sectional study of functional disabilities and perceived cognitive dysfunction in patients with major depressive disorder in South Korea: the PERFORM-K study. Psychiatry Res. 2016;239:353-361. doi:10.1016/j.psychres.2016.01.022

21. Manit S, Yee Ming M, Yen Kuang Y, et al. Cognitive dysfunction in Asian patients with depression (CogDAD): a cross-sectional study. Clin Pract Epidemiol Ment Health. 2017;13:185-199. doi:10.2174/ 1745017901713010185

22. Saragoussi D, Touya M, Haro JM, et al. Factors associated with failure to achieve remission and with relapse after remission in patients with major depressive disorder in the PERFORM study. Neuropsychiatr Dis Treat. 2017;13:2151-2165. doi:10.2147/NDT.S136343

23. Hammer-Helmich L, Haro JM, Jönsson BT, et al. Functional impairment in patients with major depressive disorder: the 2-year PERFORM study. Neuropsychiatr Dis Treat. 2018;14:239-249. doi:10.2147/NDT.S146098

24. Saragoussi D, Christensen MC, Hammer-Helmich L, Rive B, Touya M, Haro JM. Long-term follow-up on health-related quality of life in major depressive disorder: a 2-year European cohort study. Neuropsychiatr Dis Treat. 2018;14:1339-1350. doi:10.2147/NDT.S159276

25. Chokka P, Bougie J, Rampakakis E, Proulx J. Assessment in work productivity and the relationship with cognitive symptoms (AtWoRC): primary analysis from a Canadian open-label study of vortioxetine in patients with major depressive disorder (MDD). CNS Spectr. 2019;24(3):338-347. doi:10.1017/S1092852918000913

26. Chokka P, Bougie J, Proulx J, Holmegaard Tvistholm A, Ettrup A. Long-term functioning outcomes are predicted by cognitive symptoms in working patients with major depressive disorder treated with vortioxetine: results from the AtWoRC study. CNS Spectr. 2019;24 (6):616-627. doi:10.1017/S1092852919000786

27. Cambridge OR, Knight MJ, Mills N, Baune BT. The clinical relationship between cognitive impairment and psychosocial functioning in major depressive disorder: a systematic review. Psychiatry Res. 2018;269:157-171. doi:10.1016/j.psychres.2018.08.033

28. Knight MJ, Air T, Baune BT. The role of cognitive impairment in psychosocial functioning in remitted depression. J Affect Disord. 2018;235:129-134. doi:10.1016/j.jad.2018.04.051

29. Wang G, Si T-M, Li L, et al. Cognitive symptoms in major depressive disorder: associations with clinical and functional outcomes in a 6-month, non-interventional, prospective study in China. Neuropsychiatr Dis Treat. 2019;15:1723-1736. doi:10.2147/NDT.S195505

30. Hamilton M. A rating scale for depression. J Neurol Neurosurg Psychiatry. 1960;23:56-62. doi:10.1136/jnnp.23.1.56

31. Kroenke K, Spitzer RL, Williams JB. The PHQ-9: validity of a brief depression severity measure. J Gen Intern Med. 2001;16(9):606-613. doi:10.1046/j.1525-1497.2001.016009606.x

32. Fehnel SE, Forsyth BH, Dibenedetti DB, Danchenko N, François C, Brevig T. Patient-centered assessment of cognitive symptoms of depression. CNS Spectr. 2016;21(1):43-52. doi:10.1017/S10928529 13000643

33. Shi C, Wang G, Tian F, et al. Reliability and validity of Chinese version of perceived deficits questionnaire for depression in patients with MDD. Psychiatry Res. 2017;252:319-324. doi:10.1016/j. psychres.2017.03.021

34. Lam RW, Lamy FX, Danchenko N, et al. Psychometric validation of the Perceived Deficits Questionnaire-Depression (PDQ-D) instrument in US and UK respondents with major depressive disorder. Neuropsychiatr Dis Treat. 2018;14:2861-2877. doi:10.2147/NDT.S175188

35. Wechsler D. Wechsler Adult Intelligence Scale-Revised. San Antonio, TX, USA: Psychological Corporation; 1981.

36. Mahableshwarkar AR, Zajecka J, Jacobson W, Chen Y, Keefe RS. A randomized, placebo-controlled, active-reference, double-blind, flexible-dose study of the efficacy of vortioxetine on cognitive function in major depressive disorder. Neuropsychopharmacology. 2015;40(8):2025-2037. doi:10.1038/npp.2015.52 
37. McIntyre RS, Harrison J, Loft H, Jacobson W, Olsen CK. The effects of vortioxetine on cognitive function in patients with major depressive disorder: a meta-analysis of three randomized controlled trials. Int J Neuropsychopharmacol. 2016;19(10):pyw055. doi:10.1093/ijnp/ pyw055

38. EuroQol Group. EuroQol - a new facility for the measurement of health-related quality of life. Health Policy. 1990;16(3):199-208. doi:10.1016/0168-8510(90)90421-9

39. Liu GG, Wu H, Li M, Gao C, Luo N. Chinese time trade-off values for EQ-5D health states. Value Health. 2014;17(5):597-604. doi:10. 1016/j.jval.2014.05.007

40. Reilly MC, Zbrozek AS, Duke EM. The validity and reproducibility of a work productivity and activity impairment instrument. Pharmacoeconomics. 1993;4(5):353-365. doi:10.2165/00019053-19 9304050-00006

41. Sheehan DV, Harnett-Sheehan K, Raj BA. The measurement of disability. Int Clin Psychopharmacol. 1996;11(Suppl. 3):89-95. doi:10.1097/00004850-199606003-00015

42. Sheehan KH, Sheehan DV. Assessing treatment effects in clinical trials with the Discan metric of the Sheehan Disability Scale. Int Clin Psychopharmacol. 2008;23(2):70-83. doi:10.1097/YIC.0b013e3282f 2b4d6

43. R Core Team. R: a language and environment for statistical computing. R Foundation for Statistical Computing, Vienna, Austria; 2016. Available from: http://www.R-project.org/. Accessed February 18, 2020.

44. Sumiyoshi T, Watanabe K, Noto S, et al. Relationship of cognitive impairment with depressive symptoms and psychosocial function in patients with major depressive disorder: cross-sectional analysis of baseline data from PERFORM-J. J Affect Disord. 2019;258:172-178. doi:10.1016/j.jad.2019.07.064

45. McIntyre RS, Lophaven S, Olsen CK. A randomized, double-blind, placebo-controlled study of vortioxetine on cognitive function in depressed adults. Int J Neuropsychopharmacol. 2014;17(10):15 57-1567. doi:10.1017/S1461145714000546

46. Zhou T, Guan H, Yao J, Xiong X, Ma A. The quality of life in Chinese population with chronic non-communicable diseases according to EQ-5D-3L: a systematic review. Qual Life Res. 2018;27 (11):2799-2814. doi:10.1007/s11136-018-1928-y

47. Shi J-F, Huang H-Y, Guo L-W, et al. Quality-of-life and health utility scores for common cancers in China: a multicentre cross-sectional survey. Lancet. 2016;388(Spec. Iss. 1):S29. doi:10.1016/S0140-6736 (16)31956-0
48. Cha DS, Carmona NE, Subramaniapillai M, et al. Cognitive impairment as measured by the THINC-integrated tool (THINC-it): association with psychosocial function in major depressive disorder. $J$ Affect Disord. 2017;222:14-20. doi:10.1016/j.jad.2017.06.036

49. Srisurapanont M, Suttajit S, Eurviriyanukul K, Varnado P. Discrepancy between objective and subjective cognition in adults with major depressive disorder. Sci Rep. 2017;7(1):3901. doi:10. 1038/s41598-017-04353-w

50. Fava M, Mahableshwarkar AR, Jacobson W, et al. What is the overlap between subjective and objective cognitive impairments in MDD? Ann Clin Psychiatry. 2018;30(3):176-184.

51. Schwert C, Stohrer M, Aschenbrenner S, Weisbrod M, Schröder A Biased neurocognitive self-perception in depressive and in healthy persons. J Affect Disord. 2018;232:96-102. doi:10.1016/j.jad.2018. 02.031

52. Dehn LB, Beblo T. Are they just exaggerating? Differences between subjective and objective cognitive performance in patients with depression: mounting evidence and open questions. J Affect Disord. 2019;248:198-199. doi:10.1016/j.jad.2018.05.082

53. Evans-Lacko S, Knapp M. Global patterns of workplace productivity for people with depression: absenteeism and presenteeism costs across eight diverse countries. Soc Psychiatry Psychiatr Epidemiol. 2016;51(11):1525-1537. doi:10.1007/s00127-016-1278-4

54. Uribe JM, Pinto DM, Vecino-Ortiz AI, Gómez-Restrepo C, Rondón M. Presenteeism, absenteeism, and lost work productivity among depressive patients from five cities of Colombia. Value Health Reg Issues. 2017;14:15-19. doi:10.1016/j.vhri.2017.03.001

55. Beck A, Crain LA, Solberg LI, et al. The effect of depression treatment on work productivity. Am J Manag Care. 2014;20(8): e294-e301.

56. Lachaine J, Beauchemin C, Bibeau J, et al. Canadian economic impact of improved workplace productivity in patients with major depressive disorder treated with vortioxetine. CNS Spectr. 2019:1-8. doi:10.1017/S1092852919000853.

57. Baune BT, Brignone M, Larsen KG. A network meta-analysis comparing effects of various antidepressant classes on the digit symbol substitution test (DSST) as a measure of cognitive dysfunction in patients with major depressive disorder. Int J Neuropsychopharmacol. 2018;21(2):97-107. doi:10.1093/ijnp/pyx070

58. Andresen R, Caputi P, Oades LG. Do clinical outcome measures assess consumer-defined recovery? Psychiatry Res. 2010;177(3):309-317. doi:10.1016/j.psychres.2010.02.013
Neuropsychiatric Disease and Treatment

\section{Publish your work in this journal}

Neuropsychiatric Disease and Treatment is an international, peerreviewed journal of clinical therapeutics and pharmacology focusing on concise rapid reporting of clinical or pre-clinical studies on a range of neuropsychiatric and neurological disorders. This journal is indexed on PubMed Central, the 'PsycINFO' database and CAS, and is the official journal of The International Neuropsychiatric Association (INA). The manuscript management system is completely online and includes a very quick and fair peer-review system, which is all easy to use. Visit http://www.dovepress.com/testimonials.php to read real quotes from published authors. 